\title{
Comparison of In Vivo Pharmacokinetics and Pharmacodynamics of Vancomycin Products Available in Korea
}

\author{
Hee Kyung Kim ${ }^{1}$, Su-Mi Choi ${ }^{1}$, Gaeun Kang ${ }^{2}$, Kyung-Hwa Park ${ }^{1}$, Dong-Gun Lee ${ }^{3}$, Wan-Beom Park ${ }^{4}$, \\ Su-jin Rhee ${ }^{5}$, SeungHwan Lee ${ }^{5}$, Sook-In Jung ${ }^{1}$, and Hee-Chang Jang ${ }^{1}$ \\ ${ }^{1}$ Department of Infectious Diseases, Chonnam National University Medical School, Gwangju; \\ ${ }^{2}$ Division of Clinical Pharmacology, Department of Pharmacology, Chonnam National University Medical School, Gwangju; \\ ${ }^{3}$ Division of Infectious Diseases, Department of Internal Medicine, College of Medicine, The Catholic University of Korea, Seoul; \\ ${ }^{4}$ Department of Internal Medicine, Seoul National University College of Medicine, Seoul; \\ ${ }^{5}$ Department of Clinical Pharmacology and Therapeutics, Seoul National University College of Medicine and Hospital, Seoul, Korea.
}

Purpose: Few studies have been investigated the in vivo efficacy of generic vancomycin products available outside of the United States. In this study, we aimed to compare the in vivo pharmacokinetics (PK) and pharmacodynamics (PD) of five generic vancomycin products available in Korea with those of the innovator.

Materials and Methods: The in vitro vancomycin purity of each product was examined using high-pressure liquid chromatography. Single-dose PK analyses were performed using neutropenic mice. The in vivo efficacy of vancomycin products was compared with that of the innovator in dose-effect experiments ( 25 to $400 \mathrm{mg} / \mathrm{kg}$ per day) using a thigh-infection model with neutropenic mice.

Results: Generic products had a lower proportion of vancomycin B (range: 90.3-93.8\%) and a higher proportion of impurities (range: 6.2-9.7\%) than the innovator (94.5\% and 5.5\%, respectively). In an in vivo single-dose PK study, the maximum concentration $\left(\mathrm{C}_{\max }\right)$ values of each generic were lower than that of the innovator, and the geographic mean area under the curve ratios of four generics were significantly lower than that of the innovator (all $p<0.1$ ). In the thigh-infection model, the maximum efficacies of generic products reflected in maximal effect $\left(\mathrm{E}_{\max }\right)$ values were not significantly different from the innovator. However, the PD profile curves of some generic products differed significantly from that of the innovator in mice injected with a high level of Mu3 (all $p \leq 0.05$ ).

Conclusion: Some generic vancomycin products available in Korea showed inferior PK and PD profiles, especially in mice infected with hetero-vancomycin-resistant Staphylococcus aureus.

Key Words: Vancomycin, generic, original, innovator, efficacy

Received: October 25, 2019 Revised: March 10, 2020

Accepted: March 10, 2020

Co-corresponding authors: Hee-Chang Jang, MD, PhD, Department of Infectious Diseases, Chonnam National University Medical School, 42 Jebong-ro, Donggu, Gwangju 61469, Korea.

Tel: 82-62-220-6296, Fax: 82-62-225-8578, E-mail: haroc153@naver.com and Sook-In Jung, MD, PhD, Department of Infectious Diseases, Chonnam National University Medical School, 42 Jebong-ro, Dong-gu, Gwangju 61469, Korea. Tel: 82-62-220-6296, Fax: 82-62-225-8578, E-mail: sijung@chonnam.ac.kr

-The authors have no potential conflicts of interest to disclose.

(C) Copyright: Yonsei University College of Medicine 2020

This is an Open Access article distributed under the terms of the Creative Commons Attribution Non-Commercial License (https://creativecommons.org/licenses/ by-nc/4.0) which permits unrestricted non-commercial use, distribution, and reproduction in any medium, provided the original work is properly cited.

\section{INTRODUCTION}

Staphylococcus aureus is the leading cause of various community- and hospital-acquired infections, including those of the skin and soft tissue, respiratory system, blood, bone, and prosthetic joints. ${ }^{1,2}$ Vancomycin, the first glycopeptide antimicrobial agent, is an antibiotic commonly prescribed for treating life-threatening infections caused by Gram-positive bacteria, including S. aureus. ${ }^{3}$ However, in recent decades, vancomycin treatment failure has become a major clinical problem in patients with serious methicillin-resistant $S$. aureus (MRSA) infections. ${ }^{4,5}$ Several studies have suggested that both MRSA strains exhibiting increased vancomycin minimum inhibitory 
concentrations (MICs) and hetero-resistant vancomycin-intermediate $S$. aureus (h-VISA) are associated with treatment failure and mortality. ${ }^{6-8}$

Since the 1980s, the clinical use of generic medicines has increased; however, systematic evaluation of their therapeutic efficacies in comparison to those of their respective innovators is lacking. ${ }^{9,10}$ To date, no country has required a demonstration of the therapeutic equivalence of generic formulations of parenteral antibiotics. Recent studies have investigated the efficacies of generic vancomycin products in vivo ${ }^{11}$ and indicated that some generics are less efficacious than the innovators, ${ }^{12-14}$ while others are of similar efficacy. ${ }^{15-17}$

Although concerns for the in vivo therapeutic equivalence of generic vancomycin products have emerged, few studies have compared the pharmacokinetics (PK) or pharmacodynamics (PD) of vancomycin and generics thereof available outside of the US. In this study, we compared the in vivo PK and $\mathrm{PD}$ of generic vancomycin products available in Korea with those of the innovator.

\section{MATERIALS AND METHODS}

\section{Ethics}

All animal experiments were approved by the Chonnam National University Institutional Review Board (CNU IACUC$\mathrm{H}-2015-13)$. All procedures were performed in accordance with the guidelines of the Animal Care and Use Committee of Chonnam National University.

\section{Bacteria, reagents, and in vitro susceptibility tests}

The antibacterial efficacies of generic vancomycin products were investigated using $S$. aureus ATCC 29213 (vancomycin $\mathrm{MIC}=1 \mathrm{mg} / \mathrm{L}$ ) and Mu3 (h-VISA; vancomycin MIC=2 mg/L). Bacteria were cultured overnight on Mueller-Hinton agar plates. A few colonies were suspended in cation-adjusted MuellerHinton II broth and grown to mid-log phase. The bacterial suspensions were diluted to the desired concentrations and used immediately. The in vitro MIC and minimum bactericidal concentration (MBC) of each product were determined via broth microdilution following the guidelines of the Clinical and Laboratory Standards Institute. ${ }^{18}$ The MICs of the innovator and five generics were evaluated simultaneously, as were the MBCs of all products. All tests were performed in duplicate.

\section{Vancomycin products}

Vancocin CP (Eurofarma Laboratorios Ltda, Sao Paulo, Brazil), produced using the original methods of Eli Lilly and Company, was used throughout the study. Eli Lilly earlier terminated vancomycin production and sold the brand name to several manufacturers worldwide, a deal which generated changes in the Korean market. Innovex Pharma Korea (Seoul, Republic of Korea), currently Menarini Pharma Korea, then commercial- ized the Vancocin CP produced by Eurofarma Laboratorios Ltda.

Five generic vancomycin products commercially available in the Republic of Korea were evaluated: CJ-vancomycin hydrochloric acid ( $\mathrm{HCl}$ ) (CheilJedang Pharmaceuticals, Incheon, Republic of Korea); Hanomycin (Samjin Pharmaceuticals, Hwaseong, Republic of Korea); Pfizer-vancomycin (Xellia Pharmaceuticals, Copenhagen, Denmark); Dong-A vancomycin $\mathrm{HCl}$ (Dong-A Pharmaceuticals, Cheonan, Republic of Korea); and Huons-vancomycin $\mathrm{HCl}$ (BCWorld Pharmaceuticals, Yeoju, Republic of Korea). Vancomycin products were obtained from the pharmacy departments of university hospitals, not from the drug makers or sellers.

Vancomycin factor $B$ concentrations and product purity We measured vancomycin factor B concentrations and the purities of all products via high-pressure liquid chromatography (HPLC) (Shimadzu, Kyoto, Japan) at the Chonnam National Hospital Clinical Trial Center. In brief, the HPLC platform consisted of a dual solvent pump (model LC-20AD), autosampler (model SIL-20AC), column oven (model CTO-20AC), UV/VIS detector (model SPD-20A), and communication bus module (model CBM-20A). We used a LUNA C18(2) column (particle size $5 \mu \mathrm{m}, 250 \times 4.6 \mathrm{~mm}$; Phenomenex, Torrance, CA, USA) fitted with a Security Guard C18 Cartridge $(4 \times 3.0 \mathrm{~mm})$ operating at $40^{\circ} \mathrm{C}$ to separate vancomycin factor $\mathrm{B}$ from any impurities via gradient elution. The flow rate of the mobile phase containing a mixture $(50: 50 \mathrm{v} / \mathrm{v})$ of solvents A (acetonitrile) and $\mathrm{B}$ (acetonitrile with $5 \mathrm{mM} \mathrm{KH}_{2} \mathrm{PO}_{4}, \mathrm{pH} 3.0$ ) was 1 $\mathrm{mL} / \mathrm{min}$. The initial $10 \%(\mathrm{v} / \mathrm{v})$ of solvent $\mathrm{B}$ was increased to $22 \%$ (v/v) B over 15 min and then decreased back to $10 \%$ (v/v) over $6 \mathrm{~s}$. The injection volume was $20 \mu \mathrm{L}$, and the eluted products were detected at $280 \mathrm{~nm}$. Shimadzu LC solution software was used for data analysis. In preliminary work, we evaluated a vancomycin reference standard, purchased from the U.S. Pharmacopeia (USP; Rockville, MD, USA), and the impurity CDP-1, purchased from Carbosynth Ltd (Compton, Berkshire, UK).

\section{Animal experiments}

We used 6-week-old, pathogen-free, ICR female mice (weight 24-28 g) from the Institute for Cancer Research (Samtako, Osan, Republic of Korea). The animals were anesthetized by intraperitoneal injection of $100 \mu \mathrm{L}$ of phosphate-buffered saline containing $2 \mathrm{mg}$ of ketamine and $0.2 \mathrm{mg}$ of xylazine. Mice were rendered neutropenic by two intraperitoneal injections of cyclophosphamide (Sigma-Aldrich, St. Louis, MO, USA) administered 4 days $(150 \mathrm{mg} / \mathrm{kg}$ ) and 1 day $(100 \mathrm{mg} / \mathrm{kg})$ prior to $\mathrm{PK} / \mathrm{PD}$ evaluation, as described previously. ${ }^{19}$

\section{Single-dose serum PK in neutropenic mice}

Six groups of 24 neutropenic mice not infected by $S$. aureus were administered a single dose (100 mg/kg in $200 \mu \mathrm{L}$ saline) of each vancomycin product subcutaneously. Blood samples for 
PK analyses were obtained from subgroups of four animals at $0.25,1,2$, and $4 \mathrm{hr}$. At each time point, animals were anesthetized and then euthanized by cervical dislocation. Blood was collected from individual mice by cardiac puncture. ${ }^{17,20-24}$ Each animal constituted a single data point. All experiments were repeated 10 times for subgroups of each product. Serum was obtained by centrifugation of the blood sample at $10000 \times \mathrm{g}$ for $5 \mathrm{~min}$; all samples were stored at $-70^{\circ} \mathrm{C}$ prior to analysis. Vancomycin concentrations were determined via liquid chromatography (API 4000Q TRAP; AB SCIEX, Foster City, CA, USA) performed at the Chonnam National University Hospital Clinical Trial Center. PK parameters were calculated by a onecompartment analysis after determination of the antibiotic concentration at the different time points. The innovator and the five generics were evaluated simultaneously.

\section{Thigh-infection model with neutropenic mouse and in vivo $P D$}

Both thighs of neutropenic mice were inoculated with $100 \mu \mathrm{L}$ of log-phase cultures containing $10^{8}$ (high-level) or $10^{4}$ (lowlevel) S. aureus ATCC 29213 or Mu3. Two hours later, vancomycin was injected subcutaneously at 25, 50, 100, 200, or 400 $\mathrm{mg} / \mathrm{kg}$ of body weight; this was repeated three times at $6 \mathrm{~h}$ intervals. Three mice per experiment served as controls (no vancomycin). After $24 \mathrm{hr}$, animals were anesthetized and then euthanized by cervical dislocation. Then, the thighs were aseptically dissected and homogenized, and the suspensions were serially diluted and plated for bacterial counts. The innovator and the five generics were evaluated simultaneously. All experiments were repeated three times, and the data were subjected to statistical analysis.

\section{Statistical analysis}

Phoenix NLME 1.3 (Certara, St. Louis, MO, USA), a population PK modeling software, was used to fit the data to a one-compartment model exhibiting first-order absorption and elimination. The population PK parameter estimates were used to obtain the area under the curve (AUC), maximum concentration $\left(\mathrm{C}_{\max }\right)$, and half-life. Bioequivalence was considered present when the $90 \%$ confidence interval (CI) of the geometric mean ratio (Test/Reference) was $80-125 \%$. We did not round off the CI values; therefore, to pass this test, the CI had to be at least $80 \%$ and no more than $125 \%$, as required by drug regulatory agencies. ${ }^{25,26}$ A $p$ value $\leq 0.1$ was considered to indicate statistical significance in the AUC and $\mathrm{C}_{\max }$ comparisons, in line with the recommendations of drug regulatory agencies.

In terms of $\mathrm{PD}$, we calculated the net antibacterial effect (E, the dependent variable) of each drug dose (D, the independent variable) by subtracting the colony-forming units (CFU)/ $\mathrm{g}$ of the untreated control mice (at $24 \mathrm{~h}$ ) from that of mice treated for $24 \mathrm{~h}$. Since $\mathrm{E}$ is a measure of bacterial killing, $\mathrm{E}$ is negative, except when bacterial growth actually exceeds that in the untreated mice. Least-squares nonlinear regression pro- duced three PD parameters describing the dose effects. These parameters were subjected to further nonlinear regression using the Hill sigmoid model to estimate the maximal effect $\left(\mathrm{E}_{\mathrm{max}}\right)$, the effective dose needed to attain $50 \%$ of the $\mathrm{E}_{\max }\left(\mathrm{ED}_{50}\right)$, and the slope (N) for each product (SigmaPlot 10.0; Systat Software Inc., San Jose, CA, USA) according to the following equation:

$$
\mathrm{E}=\frac{\mathrm{E}_{\max } \times \text { Dose }^{\mathrm{N}}}{\mathrm{ED}_{50}^{\mathrm{N}}+\text { Dose }^{\mathrm{N}}}
$$

The secondary PD profiles reflect the exact potency of a product. We computed the in vivo doses ( $\mathrm{mg} / \mathrm{kg} / \mathrm{day}$ ) required to attain a net bacteriostatic effect (BD) and to kill the first log of bacteria (1LKD). The net bacterial growth $(\mathrm{G})$ of the untreated controls (CFU/g at $24 \mathrm{~h}-\mathrm{CFU} / \mathrm{g}$ at $0 \mathrm{~h}$ ) was used to replace E when the antibiotic prevented BD or allowed attainment of the 1LKD. The secondary PD parameters were derived using the following equations:

$$
\begin{aligned}
& \mathrm{BD}=10^{\left\{\left[\log 10\left(\frac{\mathrm{G}}{\mathrm{E}_{\max } \mathrm{G}}\right) / \mathrm{N}\right]+\log \mathrm{ED}_{50}\right\}} \\
& 1 \mathrm{LKD}=10^{\left\{\left[\log 10\left(\frac{\mathrm{G}+1}{\mathrm{E}_{\max }(\mathrm{G}+1)}\right) / \mathrm{N}\right]+\log \mathrm{ED} 50\right\}}
\end{aligned}
$$

Primary and secondary PD parameters were compared by curve-fitting analysis (GraphPad Prism 5.0; GraphPad Software Inc., San Diego, CA, USA). ${ }^{12}$ A $p$ value $\leq 0.05$ was considered to reflect statistical significance.

\section{RESULTS}

\section{In vitro susceptibility tests}

The MICs of the six vancomycin products acting against the $S$. aureus strains ATCC 29213 and Mu3 were 1 and $2 \mathrm{mg} / \mathrm{L}$, respectively. The MBCs of the six vancomycin products acting against the $S$. aureus strains ATCC 29213 and Mu3 were 2 and 4 $\mathrm{mg} / \mathrm{L}$, respectively. No differences were observed among the products.

\section{Levels of vancomycin factor $B$ and impurities}

All vancomycin products were subjected to purity testing. We measured the baseline resolutions between crystalline degradation product atropisomers (CDP-1 $\mathrm{m}$ and CDP-1 M) and vancomycin factor B (Fig. 1). A vancomycin factor B calibration curve (1-20 mg/L) was used to evaluate the linearity of the assay. In a preliminary experiment, the mean linear regression equation was $\mathrm{Y}=0.184079 \mathrm{X}+0.0107533$ (coefficient of determination $\left.\left[\mathrm{R}^{2}\right]=0.9995\right)$.

The levels of vancomycin B, all impurities, and any principal impurities were calculated as area percentages (Table 1). The generics had low proportions of vancomycin B (range: 90.3-93.8\%) and high proportions of impurities (range: 6.2$9.7 \%)$, compared with the innovator $(94.5 \%$ and $5.5 \%$, respectively). However, all products met the USP standard of con- 


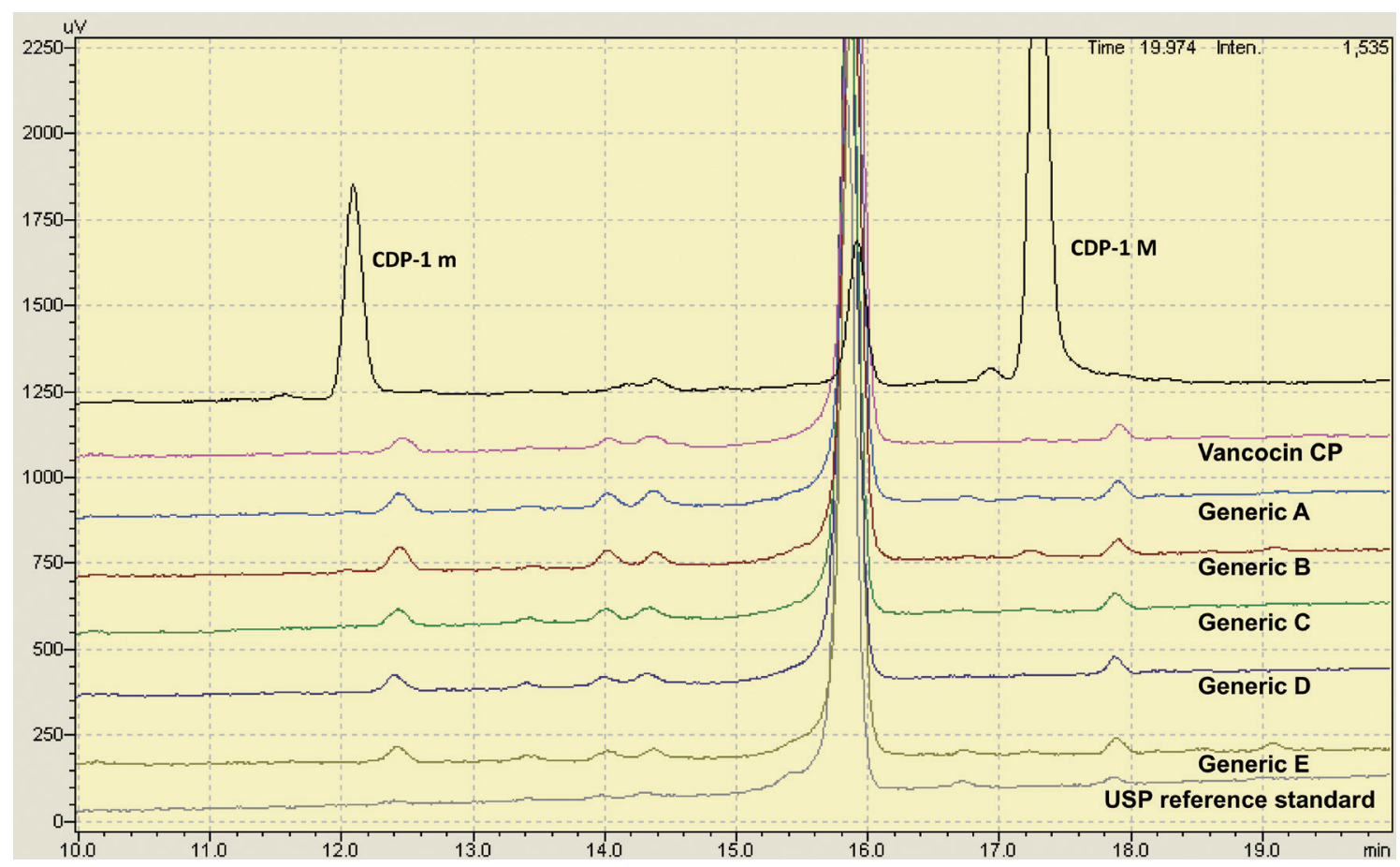

Fig. 1. Representative chromatograms of six vancomycin products using the HPLC method. The main peak just before 15.8 min is vancomycin $B$. The retention time of the vancomycin peak in the test products was consistent with the USP reference standard. HPLC, high-pressure liquid chromatography; USP, U.S. Pharmacopeia.

Table 1. Purity and Impurities of Vancomycin Products

\begin{tabular}{lccc}
\hline Product & $\begin{array}{c}\text { Vancomycin B } \\
(\%)\end{array}$ & $\begin{array}{c}\text { Total impurities } \\
(\%)\end{array}$ & $\begin{array}{c}\text { Largest impurity } \\
(\%)\end{array}$ \\
\hline Vancocin CP & 94.5 & 5.5 & 2.0 \\
Generic A & 90.7 & 9.3 & 2.7 \\
Generic B & 90.3 & 9.7 & 3.1 \\
Generic C & 92.2 & 7.8 & 2.4 \\
Generic D & 93.8 & 6.2 & 2.0 \\
Generic E & 92.9 & 7.1 & 2.1 \\
\hline
\end{tabular}

taining at least $80 \%(\mathrm{w} / \mathrm{w})$ vancomycin factor B and no more than $9 \%(w / w)$ of any impurity.

\section{In vivo single-dose serum $P K$}

The PK values in the sera of neutropenic mice after subcutaneous administration of single doses of each of the six tested products $(100 \mathrm{mg} / \mathrm{kg})$ are shown in Table 2 . The $\mathrm{C}_{\max }$ values of each generic were lower than that of the innovator (all $p<0.1$ ), and the $90 \%$ CIs of all generic products failed to fall within the $80-$ $125 \%$ range. The geographic mean AUC ratios of generics A, B, $\mathrm{C}$, and E were significantly lower than that of the innovator (all $p<0.1$ ), and the $90 \%$ CIs of these four products did not fall within the $80-125 \%$ range.

\section{In vivo PD data derived using the neutropenic mouse thigh-infection model}

The dose effects of the generics were compared with that of the innovator (25-400 $\mathrm{mg} / \mathrm{kg}$ per day) using a neutropenic mouse thigh-infection model injected with low and high levels of S. aureus strains ATCC29213 and Mu3. All PD parameters are shown in Table 3 and Fig. 2. The $\mathrm{E}_{\max }$ values of all six products did not differ significantly (Table 3 ). The PD profile curves of generics A, C, and D did not differ from that of innovator in mice injected with low and high levels of ATCC 29213 and low level of Mu3; however, they did differ from that of the innovator in mice injected with a high level of Mu3 (all $p \leq 0.05$ ) (Fig. 2, Table 3). The PD profile curves of generic E differed significantly from that of the innovator in mice injected with low and high levels of ATCC 29213 ( $p \leq 0.05$ ) (Table 3).

We also compared the secondary $\mathrm{PD}$ parameters $\left(\mathrm{ED}_{50}, \mathrm{BD}\right.$, and 1LKD) of the generics with those of the innovator. The $\mathrm{ED}_{50}$ and 1LKD were similar between the products. However, the BDs of generics B, C, and E differed significantly from that of the innovator in mice injected with low levels of ATCC 29213 (all $p<0.05$ ) (Table 3). The BDs of generics B and C also differed significantly from that of the innovator in mice injected with low levels of Mu3 (both $p<0.05$ ) (Table 3).

\section{DISCUSSION}

We found that the vancomycin generics available in Korea exhibit PK profiles inferior to that of the innovator. The $\mathrm{E}_{\max } \mathrm{val}-$ ues did not differ significantly; however, the PD profile curves of some generic products in high inoculum h-VISA infection 
Hee Kyung Kim, et al.

Table 2. Single-Dose Pharmacokinetics of Six Vancomycin Products after Subcutaneous Administration of $100 \mathrm{mg} / \mathrm{kg}$ in Neutropenic Mice

\begin{tabular}{lccccccc}
\hline Product & $\mathbf{T}_{\mathbf{1} / \mathbf{2}}(\mathbf{h})$ & $\mathbf{C}_{\max }(\mathbf{m g} / \mathbf{L})$ & $\mathbf{G M R}(\mathbf{9 0} \% \mathbf{C I})$ & $\boldsymbol{p}$ value & AUC $(\mathbf{m g} * \mathbf{h} / \mathbf{L})$ & $\mathbf{G M R}(\mathbf{9 0} \% \mathbf{C I})$ & $\boldsymbol{p}$ value \\
\hline Vancocin CP & 0.83 & 86.92 & Reference & Reference & 135.93 & Reference & Reference \\
Generic A & 0.56 & 68.66 & $75.14(65.84-85.76)$ & 0.001 & 100.73 & $78.88(69.75-89.22)$ & 0.002 \\
Generic B & 0.59 & 71.83 & $75.16(65.86-85.78)$ & 0.001 & 102.30 & $82.33(72.80-93.12)$ & 0.011 \\
Generic C & 0.71 & 79.05 & $81.65(71.54-93.18)$ & 0.013 & 114.40 & $87.97(77.78-99.50)$ & 0.087 \\
Generic D & 0.70 & 77.95 & $84.77(74.28-96.74)$ & 0.041 & 114.93 & $89.57(79.20-101.31)$ & 0.140 \\
Generic E & 0.61 & 74.71 & $76.92(67.40-87.79)$ & 0.001 & 104.66 & $86.32(76.32-97.63)$ & 0.050 \\
\hline
\end{tabular}

$\mathrm{T}_{1 / 2}$, half-life; $\mathrm{C}_{\max }$, maximum serum concentration; $\mathrm{AUC}$, area under the curve; $\mathrm{GMR}$, geometric mean ratio of test/reference; $\mathrm{Cl}$, confidence interval.

differed significantly from that of the innovator. About 30 vancomycin products are available in Korea, of which six were used in this study. Vancomycin accounts for $11 \%$ of the innovator (vancocin $\mathrm{CP}$ ), $16 \%$ of generic $\mathrm{A}, 6 \%$ of generic $\mathrm{B}$, and about $1 \%$ of generics C, D, and E (from the year of the study, 2013-2016).

Antibiotics are produced via chemical synthesis or fermentation, with those produced by the former method generally being purer. When antibiotics are produced by fermentation, the desired component, structurally similar components, and degradation products often coexist, despite the use of several purification steps. Thus, the quality of generics (including vancomycin) has been a concern, and the contents of the preparations vary depending on the refining processes used. ${ }^{27-29} \mathrm{Impu}-$ rities that are structurally similar to vancomycin, but exhibit reduced activities, include monodechloro-vancomycin, dimethyl-vancomycin, agluco-vancomycin, desvancosaminyl-vancomycin, desamino-vancomycin B, CDP-1M, and CDP-1m. All competitively inhibit vancomycin B activity.

We found that the generics had 0.7-4.2\% less vancomycin B and more impurities, compared with the innovator, although all products surpassed the criteria of the USP. We explored whether these differences affected in vivo PK and PD parameters. The single-dose PK parameters of the generics were inferior to those of the innovator, and generics exhibiting reduced $\mathrm{C}_{\max }$ values and AUCs in vivo tended to have a lower vancomycin factor B content. Our results are in contrast to those of previous studies ${ }^{12,17}$ that found no significant differences in PK parameters, possibly explained by the vancomycin doses used (10-50 mg/kg). We injected vancomycin at $100 \mathrm{mg} / \mathrm{kg}$, as suggested by a previous study. ${ }^{19} \mathrm{~A}$ mouse vancomycin dose of 100 $\mathrm{mg} / \mathrm{kg}$ is equivalent to a human dose of $8.1 \mathrm{mg} / \mathrm{kg}$. ${ }^{30}$ The usual therapeutic dose of vancomycin used in patients is 7.5 to $15 \mathrm{mg}$ / $\mathrm{kg} /$ dose (500-1000 mg/dose). It is known that a serum trough concentration of $10-15 \mu \mathrm{g} / \mathrm{mL}$ of vancomycin is recommended for the treatment of serious $S$. aureus infections, and some experts recommend $15-20 \mu \mathrm{g} / \mathrm{mL}$. There are several reports that show a correlation between the AUC and the outcome of MRSA infections. For this reason, a physician must test the serum trough concentration of vancomycin and adjust doses appropriately. ${ }^{31}$ We found that the in vivo $\mathrm{C}_{\max }$ values and the AUCs of the generics were inferior to those of the innovator, suggesting that $\mathrm{C}_{\max }$ and AUC may be reduced in some patients treated with generics, despite the purity profiles meet- ing the USP criterion. Clinically speaking, PK parameters, including AUC/MIC ratio, can affect the treatment outcomes of MRSA infections; thus, further human studies are needed to evaluate the associations between the levels of vancomycin factor B in generics and serum concentrations in patients.

In our experiments, vancomycin treatment was given subcutaneously every $6 \mathrm{~h}$, in accordance with previous PD models with mice; ${ }^{17,19}$ however, some experts recommend a q3h dosing schedule of vancomycin for animal experiments ${ }^{28}$ to reach the required $\mathrm{T}>\mathrm{MIC}$ of vancomycin for maximum efficacy and not to undermine the power to detect significant differences in in vivo efficacy between the products. We found that the in vivo half-lives of all products tested were $0.56-0.83$ h; no significant differences were observed, consistent with a previous study using injection of $100 \mathrm{mg} / \mathrm{kg}$ vancomycin. ${ }^{19,24}$ Some previous studies using lower drug dose injections reported shorter half-lives: the half-life of vancomycin is dependent on the dose injected, increasing with an increase in dose. ${ }^{19}$

The in vivo efficacies of vancomycin generics have been evaluated in previous studies. Vesga, et al. ${ }^{12}$ showed that one generic had a low in vivo $\mathrm{E}_{\max }$ and anti-h-VISA action in mice compared with the innovator. ${ }^{13}$ However, other investigators found no in vivo differences between generics and the innovator. ${ }^{15-17}$ Tattevin, et al. ${ }^{32}$ and Louie, et al. ${ }^{17}$ compared the activities of six injected vancomycin generics in a rabbit model and a neutropenic mouse thigh-infection model, and no differences in efficacy were noted. We found that the $\mathrm{E}_{\max }$ of six vancomycin products did not differ when high and low levels of ATCC 29213 and Mu3 were injected into mice. However, the levels of generics, compared with the innovator, required to achieve bacteriostasis in mice administered low levels of ATCC 29213 and Mu3 were higher than that of the innovator, and the generics exhibited inferior dose-effect relationships in the high-level h-VISA model of infection. Meanwhile, we did note that $\mathrm{E}_{\mathrm{max}}$, slope $(\mathrm{N})$, and $\mathrm{ED}_{50}$ did not differ significantly between the innovator and the generic drugs, although the PD profile curves of some generic products in high inoculum hVISA infection differed significantly from that of the innovator. The results seem to suggest that the differences in efficacy are primarily due to PK differences and possibly due to the low proportion of vancomycin B in generic products, compared to the innovator. While our data do suggest that the maximum drug effects do not differ among vancomycin generics and the 


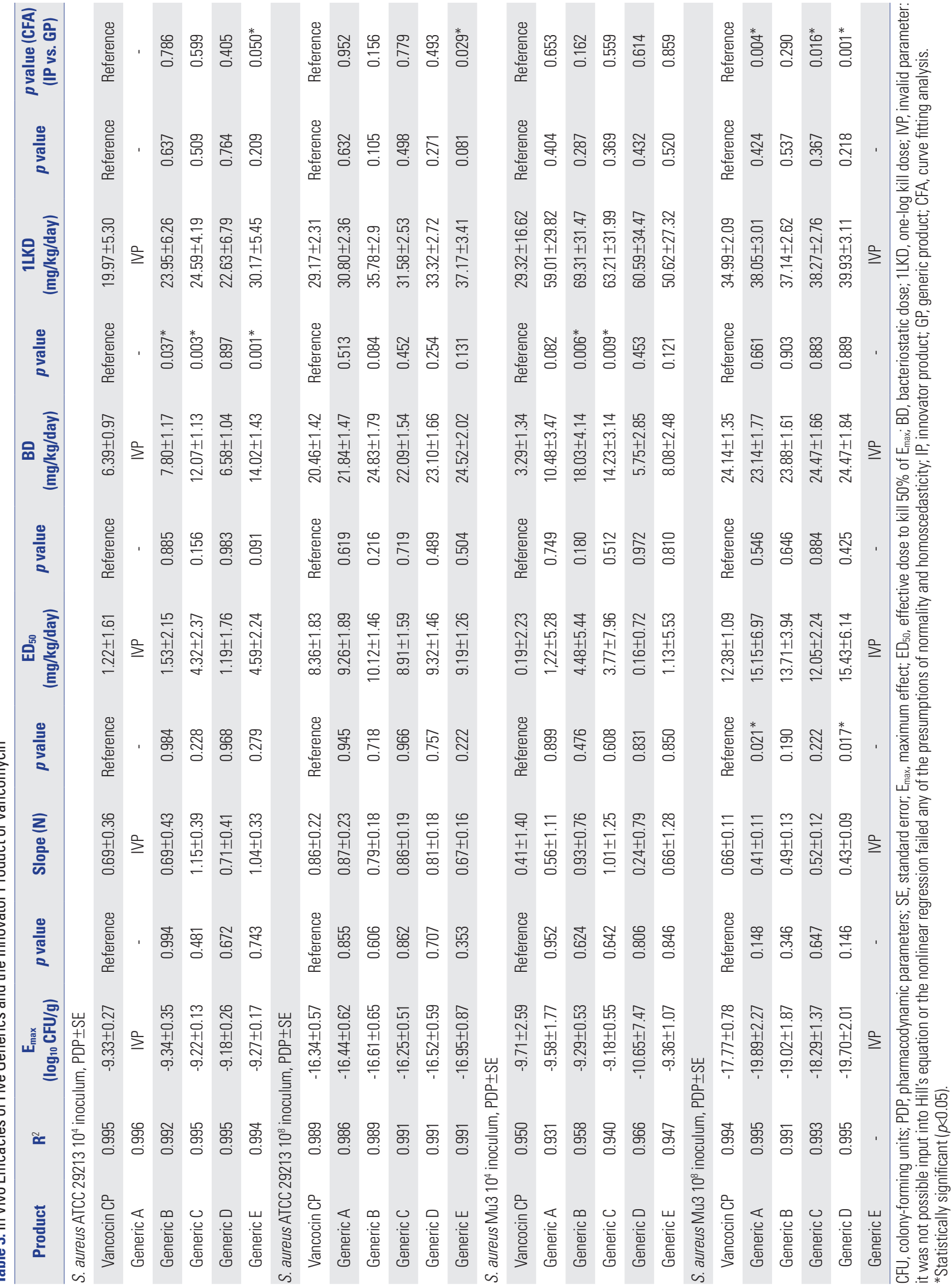



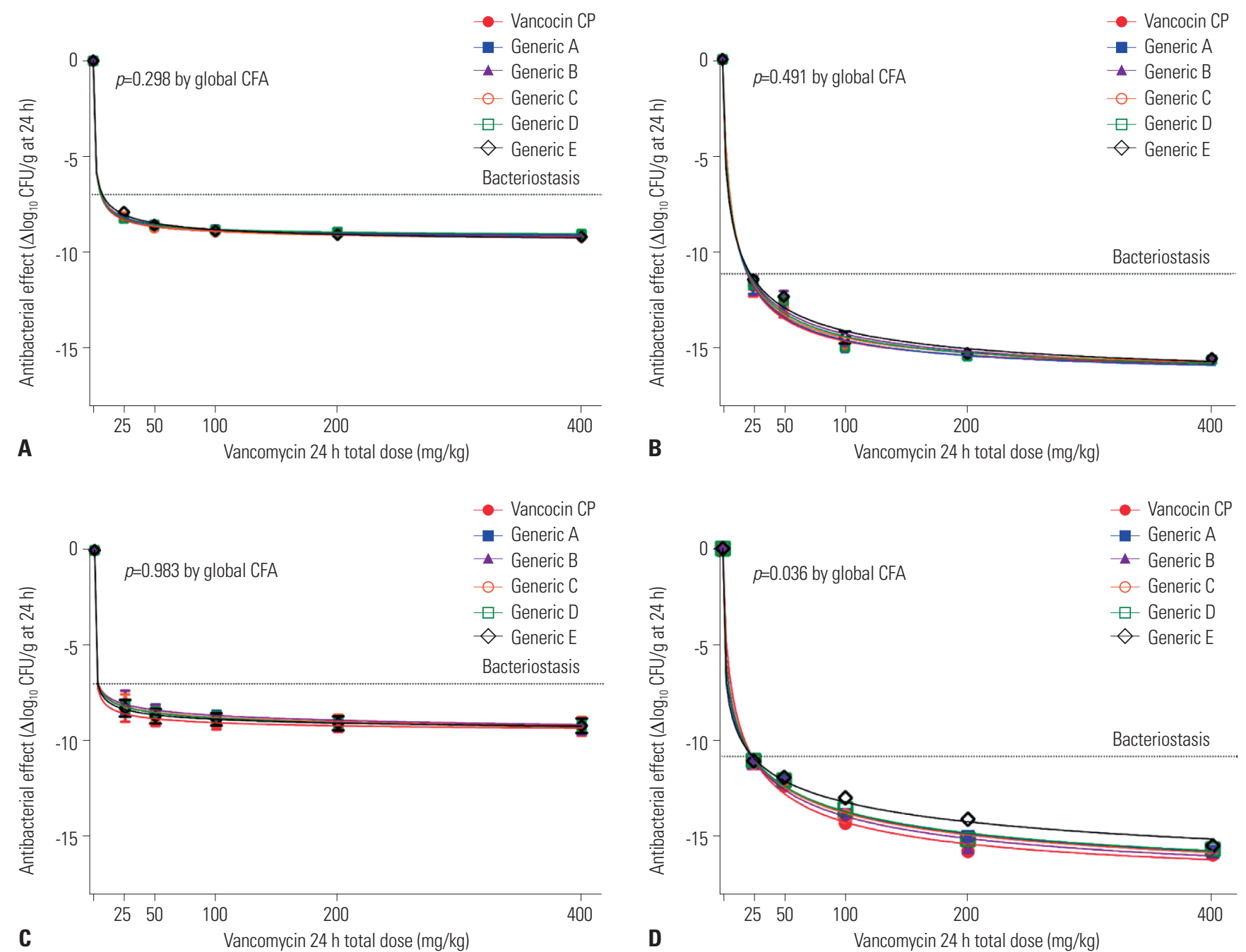

Fig. 2. Dose-response relationship of the innovator and five generic products. Five generic vancomycin products were compared with the innovator in dose-effect experiments ( 25 to $400 \mathrm{mg} / \mathrm{kg}$ per day) using a neutropenic mice thigh-infection model by injecting ATCC 29213 at low (A) and high (B) inocula ( $\sim$ and $\sim 8 \log _{10}$ CFU per thigh) and Mu3 at low (C) and high (D) inocula. Innovator and five generics were analyzed by non-linear regression fitting Hill's sigmoid model. Each data point represents the mean CFU/g of both thighs from a single mouse and three experiments. CFU, colony-forming units; CFA, curve fitting analysis.

innovator, when using some generic products, higher doses may be required, especially in patients with h-VISA infections. Further human PD studies are required to determine whether our mouse data can be replicated in humans with MRSA or hVISA infections.

\section{ACKNOWLEDGEMENTS}

This study was supported by research funds from Chonnam National University Hospital (CRI13032-1). The provider of financial support had no role in the study design, data collection and analysis, decision to publish, or preparation of the manuscript.

\section{AUTHOR CONTRIBUTIONS}

Conceptualization: Hee-Chang Jang and Don-Gun Lee. Data cura- tion: Hee Kyung Kim and Su-Mi Choi. Formal analysis: Gaeun Kang, Su-jin Rhee, and SeungHwan Lee. Funding acquisition: Hee-Chang Jang and Sook-In Jung. Investigation: Hee-Chang Jang and Hee Kyung Kim. Methodology: Hee-Chang Jang and Hee Kyung Kim. Project administration: Hee-Chang Jang and Hee Kyung Kim. Resources: Hee-Chang Jang, Kyung-Hwa Park, and Wan-Beom Park. Software: Hee-Chang Jang, Gaeun Kang, Su-jin Rhee, and SeungHwan Lee. Supervision: Hee-Chang Jang and Sook-In Jung. Validation: Hee-Chang Jang, Gaeun Kang, Su-jin Rhee, and SeungHwan Lee. Visualization: Hee-Chang Jang, Hee Kyung Kim, Gaeun Kang, Su-jin Rhee, and SeungHwan Lee. Writing_original draft: Hee-Chang Jang, Hee Kyung Kim, and Su-jin Rhee. Writing_review \& editing: HeeChang Jang, Hee Kyung Kim, and Su-jin Rhee. Approval of final manuscript: all authors.

\section{ORCID iDs}

Hee Kyung Kim Su-Mi Choi https://orcid.org/0000-0001-7043-0426

https://orcid.org/0000-0002-2417-262X 
Gaeun Kang Kyung-Hwa Park Dong-Gun Lee Wan-Beom Park Su-jin Rhee SeungHwan Lee Sook-In Jung Hee-Chang Jang

https://orcid.org/0000-0001-9841-1139 https://orcid.org/0000-0003-2836-6963 https://orcid.org/0000-0003-4655-0641 https://orcid.org/0000-0003-0022-9625 https://orcid.org/0000-0002-4010-0837 https://orcid.org/0000-0002-1713-9194 https://orcid.org/0000-0002-1577-678X https://orcid.org/0000-0002-3407-8493

\section{REFERENCES}

1. Lowy FD. Staphylococcus aureus infections. N Engl J Med 1998; 339:520-32.

2. Jang HC, Choi OJ, Kim GS, Jang MO, Kang SJ, Jung SI, et al. Active surveillance of the trachea or throat for MRSA is more sensitive than nasal surveillance and a better predictor of MRSA infections among patients in intensive care. PLoS One 2014;9:e99192.

3. Liu C, Bayer A, Cosgrove SE, Daum RS, Fridkin SK, Gorwitz RJ, et al. Clinical practice guidelines by the infectious diseases society of america for the treatment of methicillin-resistant Staphylococcus aureus infections in adults and children. Clin Infect Dis 2011; 52:e18-55.

4. Jang HC, Kim SH, Kim KH, Kim CJ, Lee S, Song KH, et al. Salvage treatment for persistent methicillin-resistant Staphylococcus aureus bacteremia: efficacy of linezolid with or without carbapenem. Clin Infect Dis 2009;49:395-401.

5. Jang HC, Kang SJ, Choi SM, Park KH, Shin JH, Choy HE, et al. Difference in agr dysfunction and reduced vancomycin susceptibility between MRSA bacteremia involving SCCmec types IV/IVa and I-III. PLoS One 2012;7:e49136.

6. Lodise TP Jr, Gotfried M, Barriere S, Drusano GL. Telavancin penetration into human epithelial lining fluid determined by population pharmacokinetic modeling and Monte Carlo simulation. Antimicrob Agents Chemother 2008;52:2300-4.

7. van Hal SJ, Lodise TP, Paterson DL. The clinical significance of vancomycin minimum inhibitory concentration in Staphylococcus aureus infections: a systematic review and meta-analysis. Clin Infect Dis 2012;54:755-71.

8. Appelbaum PC. Reduced glycopeptide susceptibility in methicillin-resistant Staphylococcus aureus (MRSA). Int J Antimicrob Agents 2007;30:398-408.

9. Henry D, Lexchin J. The pharmaceutical industry as a medicines provider. Lancet 2002;360:1590-5.

10. Kirking DM, Ascione FJ, Gaither CA, Welage LS. Economics and structure of the generic pharmaceutical industry. J Am Pharm Assoc (Wash) 2001;41:578-84.

11. Tattevin P, Crémieux AC, Rabaud C, Gauzit R. Efficacy and quality of antibacterial generic products approved for human use: a systematic review. Clin Infect Dis 2014;58:458-69.

12. Vesga O, Agudelo M, Salazar BE, Rodriguez CA, Zuluaga AF. Generic vancomycin products fail in vivo despite being pharmaceutical equivalents of the innovator. Antimicrob Agents Chemother 2010;54:3271-9.

13. Rodriguez CA, Agudelo M, Zuluaga AF, Vesga O. Generic vancomycin enriches resistant subpopulations of Staphylococcus aureus after exposure in a neutropenic mouse thigh infection model. Antimicrob Agents Chemother 2012;56:243-7.

14. Agudelo M, Rodriguez CA, Gonzalez JM, Zuluaga AF, Vesga O. Some generic of vancomycin prescribed in the United States fail in vivo despite pharmaceutical equivalence (PE) and bioequivalence (BE) demonstrated by the FDA. [abstract A-1323]. Poster session presented at the 54th Interscience Conference on Antimi- crobial Agents and Chemotherapy (Washington, D.C.); 2014 Sep 5-9; Washington DC.

15. Nambiar S, Madurawe RD, Zuk SM, Khan SR, Ellison CD, Faustino PJ, et al. Product quality of parenteral vancomycin products in the United States. Antimicrob Agents Chemother 2012;56:2819-23.

16. Hadwiger ME, Sommers CD, Mans DJ, Patel V, Boyne MT 2nd. Quality assessment of U.S. marketplace vancomycin for injection products using high-resolution liquid chromatography-mass spectrometry and potency assays. Antimicrob Agents Chemother 2012;56:2824-30.

17. Louie A, Boyne MT 2nd, Patel V, Huntley C, Liu W, Fikes S, et al. Pharmacodynamic evaluation of the activities of six parenteral vancomycin products available in the United States. Antimicrob Agents Chemother 2015;59:622-32.

18. Clinical and Laboratory Standards Institute. Methods for dilution antimicrobial susceptibility tests for bacteria that grow aerobically; approved standard. 9th ed. CLSI document M07-A9. Wayne, PA: Clinical and Laboratory Standards Institute; 2012.

19. Lee DG, Murakami Y, Andes DR, Craig WA. Inoculum effects of ceftobiprole, daptomycin, linezolid, and vancomycin with Staphylococcus aureus and Streptococcus pneumoniae at inocula of 10(5) and 10(7) CFU injected into opposite thighs of neutropenic mice. Antimicrob Agents Chemother 2013;57:1434-41.

20. Shin HH, Han S, Yim DS, Lee DG, Park C, Kim SH, et al. Efficacy of vancomycin against Staphylococcus aureus according to inoculum size in a neutropenic mouse infection model. Infect Chemother 2011;43:251-7.

21. Pachón-Ibáñez ME, Ribes S, Domínguez MA, Fernández R, Tubau F, Ariza J, et al. Efficacy of fosfomycin and its combination with linezolid, vancomycin and imipenem in an experimental peritonitis model caused by a Staphylococcus aureus strain with reduced susceptibility to vancomycin. Eur J Clin Microbiol Infect Dis 2011;30:89-95.

22. Peetermans WE, Hoogeterp JJ, Hazekamp-van Dokkum AM, van den Broek P, Mattie H. Antistaphylococcal activities of teicoplanin and vancomycin in vitro and in an experimental infection. Antimicrob Agents Chemother 1990;34:1869-74.

23. Nagano R, Shibata K, Naito T, Fuse A, Asano K, Hashizume T, et al. Therapeutic efficacy of BO-3482, a novel dithiocarbamate carbapenem, in mice infected with methicillin-resistant Staphylococcus aureus. Antimicrob Agents Chemother 1997;41:2278-81.

24. Reyes N, Skinner R, Benton BM, Krause KM, Shelton J, Obedencio GP, et al. Efficacy of telavancin in a murine model of bacteraemia induced by methicillin-resistant Staphylococcus aureus. J Antimicrob Chemother 2006;58:462-5.

25. US Food and Drug Administration, Center for Drug Evaluation and Research. Guidance for industry: bioavailability and bioequivalence studies submitted in NDAs or INDs-General considerations. Rockville, MD: Food and Drug Administration; 2014.

26. European Medicines Agency, Committee for Medicinal Products for Human Use. Guideline on the investigation of bioequivalence. London: European Medicines Agency; 2010.

27. Agudelo M, Vesga O. Therapeutic equivalence requires pharmaceutical, pharmacokinetic, and pharmacodynamic identities: true bioequivalence of a generic product of intravenous metronidazole. Antimicrob Agents Chemother 2012;56:2659-65.

28. Rodriguez CA, Agudelo M, Gonzalez JM, Vesga O, Zuluaga AF. An optimized mouse thigh infection model for enterococci and its impact on antimicrobial pharmacodynamics. Antimicrob Agents Chemother 2015;59:233-8.

29. Rodriguez CA, Agudelo M, Zuluaga AF, Vesga O. In vivo pharmacodynamics of piperacillin/tazobactam: implications for antimicrobial efficacy and resistance suppression with innovator and 
generic products. Int J Antimicrob Agents 2017;49:189-97.

30. Nair AB, Jacob S. A simple practice guide for dose conversion between animals and human. J Basic Clin Pharm 2016;7:27-31.

31. Holmes NE, Turnidge JD, Munckhof WJ, Robinson JO, Korman TM, O'Sullivan MV, et al. Vancomycin AUC/MIC ratio and 30-day mortality in patients with Staphylococcus aureus bacteremia. An- timicrob Agents Chemother 2013;57:1654-63.

32. Tattevin P, Saleh-Mghir A, Davido B, Ghout I, Massias L, Garcia de la Maria C, et al. Comparison of six generic vancomycin products for treatment of methicillin-resistant Staphylococcus aureus experimental endocarditis in rabbits. Antimicrob Agents Chemother 2013;57:1157-62. 